Volume 9, No.4, July - August 2020

International Journal of Advanced Trends in Computer Science and Engineering

Available Online at http://www.warse.org/IJATCSE/static/pdf/file/ijatcse219942020.pdf

https://doi.org/10.30534/ijatcse/2020/219942020

\title{
Performance Evaluation on Single-Input Single-Output and Multiple-Input Single-Output Technique in Visible Light Communication
}

\author{
Norharyati Harum ${ }^{1}$, Wahidah Md Shah ${ }^{2}$, Haniza N. ${ }^{3}$, Nurul Azma Zakaria ${ }^{4}$ and Zaheera Zainal Abidin ${ }^{5}$ \\ Information Security Forensics and Computer Networking (INFORSNET) Research Group, \\ Computer Networking, Center for Advanced Computing Technology (C-ACT), Universiti Teknikal Malaysia \\ Melaka, Malaysia, ${ }^{1}$ norharyati@utem.edu.my, ${ }^{2}$ wahidah@utem.edu.my, ${ }^{3}$ haniza@utem.edu.my, \\ ${ }^{4}$ azma@utem.edu.my, \\ ${ }^{5}$ Optimization Modelling Analytic and Simulation (OPTIMAS) Research Group, \\ Computer Networking, Center for Advanced Computing Technology (C-ACT), Universiti Teknikal Malaysia \\ Melaka, Malaysia, zaheera@utem.edu.my
}

\begin{abstract}
Visible Light Communication has been pointed as one of promising technique to replace radio frequency transmission for indoor environment due to its capability to provide a high data rate transmission. In VLC, a signal is transmitted using light produced by an LED at the transmitter and received by photodiode at receiver side. MIMO is one of the techniques that are used in wireless transmission to enhance transmission performance instead of using SISO transmission, where SISO refers to single transmitter antenna with single receiver antenna, while MIMO refers to multiple transmitter antenna and multiple receiver antenna. In VLC, SISO refers to single LED and single detector, where MIMO refers to multiple LED and multiple detectors. Besides, in VLC another technique that can be used is MISO, while multiple LED in transmitter and single detector in receiver. In this project, we evaluate performance of SISO and MISO in VLC within an indoor environment using Matlab. The performance evaluation criteria that we evaluate are based on area incidence angle, LED coverage, distance between lights and total received power
\end{abstract}

Key words : VLC, SISO, MIMO, LED communication

\section{INTRODUCTION}

Visible Light Communications (VLC) or Light Emmiting Diode (LED) communication is a wireless optical communication, that has been recognized as one of the promising technology that can provide a high speed data transmission particularly in indoor environment [1]-[3]. Due to exhausted Radio Frequency (RF) spectrum that caused by the expansion of network traffic, the need for preeminent bandwidth and spectral relief is debunked [4]. Hence, VLC can be used as a new wireless transmission method to replace
RF. VLC is predicted will become a premising wireless transmission technique in future due to its capability to provide high data rate transmission, particularly in outdoor. Eventhough both RF and VLC utilize electromagnetic waves, both inherent totally different properties. Visible light doesn't interfere with electronic devices as RF waves do. Therefore, VLC is suitable to be used for applications that are sensitive to electronic devices. A recent study conducted by ABI shows that almost $70 \%$ of information traffic is anticipated to originate from indoor users. Nevertheless, the only downside to RF is that the degradation of signal propagation. Thus, the supposed user data rate will be limited because of the attenuated propagation. As a results to this issue, VLC will offer the specified coverage considering utmost the indoor environments are illuminated. Links are also kept unbroken confidential due to the visible light being unable to get through the walls.

According to a study conducted by Cevik, the basic VLC configuration is consisted of Tx with the use of LEDs, photodetectors as Rxs, modulation of data to optics and the optical communication channel [5]. Photodetectors are the entity in the VLC system by absorbing the photons and converting it into electrical energy. The study also claimed that photodiodes are the most preferred devices as a photodetectors due to them having a small size, high sensitivity and fast response. However, the main important thing to be considered during the design and modelling stages of VLC system is the localization status of the Tx and Rx pair, which mainly defines how the signal is transmitted. The first method is whether the channel between $\mathrm{Tx}$ and $\mathrm{Rx}$ is line-of-sight (LOS) channel, where Tx-Rx is set directed to a specific point or Non-LOS where Tx and Rx are blocked with certain object, thus simply not pointed at a specific point. The LOS channel could achieve high power efficiency compared to non-LOS where the signals arrived at different time at $\mathrm{Rx}$ 
due to it being reflect by surface or object during the transmission. In the other words, there are three main components of VLC, which include transmitter (Tx), receiver $(\mathrm{Rx})$ and channel. Tx job is to convert digital data into visible light as the Rx converts the incoming light into electric signal using a photo diode. In Tx, LED is used to transmit signal, and the LED transmit power and received power at Rx change depends on LED view angle.

On the other hand, Single Input Single Output (SISO) and Multiple Input Multiple Output (MIMO) have been recognized as one of the promising technique to increase performance of RF based transmission of 5G technology. SISO used in RF refers to one antenna at Tx and one antenna at Rx. Meanwhile, MIMO is a multiple input and multiple output where both $\mathrm{Tx}$ and $\mathrm{Rx}$ use multiple antennas. Researchers has pointed out that SISO is one of the simplest as well as affordable method to be carry out compared to MIMO since MIMO requires more antennas which makes it quite complex to be implemented [6]. Still, it was revealed that MIMO could help with traffic capacity involving bottlenecks in the future. The comparison results of SISO, MISO and MIMO in rural, urban and high speed environments prove that MIMO offer maximum stability compared to SISO model [6]-[8].

Similar to RF system, VLC also suffers multipath distortion. A research study was conducted on the impact of multipath reflections on the performance of indoor VLC. The study uses LED as the transmitter and a photodiode (PD) at the receiver. Based on the study, in analysing the position performance the line-of-sights (LOS) channel have been observed without considering the production of multipath reflections. With the transmitter is a wide-beam LED source, and whereas the receiver received the data reflected from room surfaces, multipath reflections occurs due the transmitted signals being bounces off walls, ceiling and floor [9]. Since the MIMO system has been proved as a promising technique to combat multipath fading effect, it is also could help in optimizing VLC performance. For VLC SISO refers to single LED at $\mathrm{Tx}$ and single detector at receiver while MIMO refers to multiple LED at Tx and multiple detector at $\mathrm{Rx}$.

In this study, effect of implementing SISO and MISO in VLC has been discussed. MISO refers to multiple LED at Tx with single detector at Rx. The simulation design and performance evaluation have been done using MATLAB. The performance evaluation and comparison is done based on total received power and LER signal coverage at Rx based on LED viewing angle.

This paper will be organized as follows: The next section describes the methodology used to evaluate the performance of the SISO and MISO in LED communications. Section III introduces simulation design used in performance evaluation of LED communications. Sections IV discusses on result from simulation that has been designed. Finally, Section V presents our conclusions.

\section{METHODOLOGY}

This study is a simulation-based research, where research phases consist of research question, model design, model building, and findings.

- Research question - For the most part, a research question was identified that is appropriate for study by simulation. In this case, every single related issue regarding the VLC concentration its performance degradation cause from multipath distortion and interference. Then, the technique that can increase the performance in Visible Light Communication, identifying the design into simulation platform and the evaluation will evaluation will be discussed.

- Model Design - After identifying the suitable research question, a model design is sketched based on the specification of the target to be modelled in the simulation and selection of an appropriate simulation method.

- Model building - In this phase, a simulation platform is built using the design explained in the previous phase. A suitable packaging should also be incorporated together in order for it to work well according to our designated simulation platform.

- Findings and conclusions - The findings and conclusions should be formulated in response to the research questions and the results should be disseminated. The final project which is the simulation output should be evaluated whether applying the SISO and MISO technique will improve the performance in VLC.

\section{SIMULATION DESIGN}

In this project, the simulation for indoor environment is considered. The room size is $5 \mathrm{~m} \times 5 \mathrm{~m} \times 3 \mathrm{~m}$. Four white LED lamp that are equally placed and installed on the ceiling of the room. Simulation parameters of the model design is shown in Table. LED allocation and the corresponding term that is designed in the simulation are shown in Figure 1, Table 1 and Table 2 .

Table 1: Simulation parameters in VLC system

\begin{tabular}{|c|c|}
\hline Term & symbol \\
\hline Distance between LEDs & $\mathbf{a}$ \\
\hline $\begin{array}{c}\text { Radius of coverage of each LED on Rx } \\
\text { plane }\end{array}$ & $\mathbf{r}$ \\
\hline Half angle of each LED & $\boldsymbol{\theta}$ \\
\hline Rooms dimension & $\boldsymbol{L} \times \boldsymbol{R} \times \boldsymbol{H}$ \\
\hline Height of Rx from ground & $\boldsymbol{h}$ \\
\hline
\end{tabular}


Figure 2 and Figure 3 show simulation design for one LED and multiple LEDs. The one LED diagram is used for SISO performance evaluation while multiple LEDs diagram is used for MISO performance evaluation.

Table 2: Simulation parameters in VLC system

\begin{tabular}{|c|c|}
\hline Parameter & Value \\
\hline Power emitted by LED (mW) & $30 \mathrm{E}-3^{*}$ \\
\hline Max LED viewing angle (led_va) & 15 \\
\hline $\begin{array}{c}\text { Field of view of PIN photodiode } \\
\text { (PD_FOV/2) }\end{array}$ & 20 \\
\hline Detector area (ARX) & $.78 \mathrm{E}-4$ \\
\hline Distance between Tx and Rx (m) & 1.5 \\
\hline Height of room & 3.0 \\
\hline Photodiode responsivity & 0.55 \\
\hline Noise power of ambient light & $0.07 \mathrm{E}-6 /$ response \\
\hline Data rate & 115200 \\
\hline Noise bandwidth factor & 0.562 \\
\hline Amplifier bandwidth & $4.5 \mathrm{E} 6$ \\
\hline Amplifier noise current & $5 \mathrm{E}-12 * \mathrm{Ba}$ \\
\hline
\end{tabular}

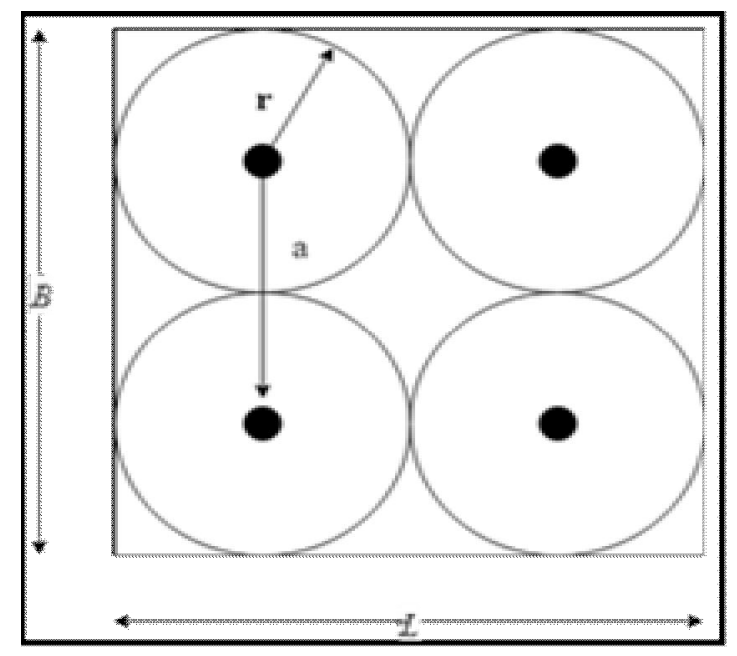

Figure 1: LED allocation for Simulation

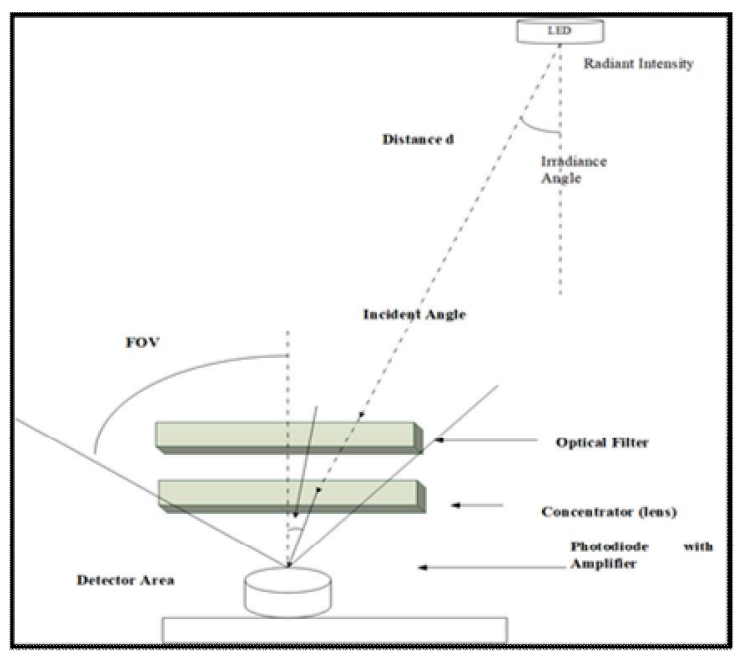

Figure 2: VLC Model with one LED in indoor environment

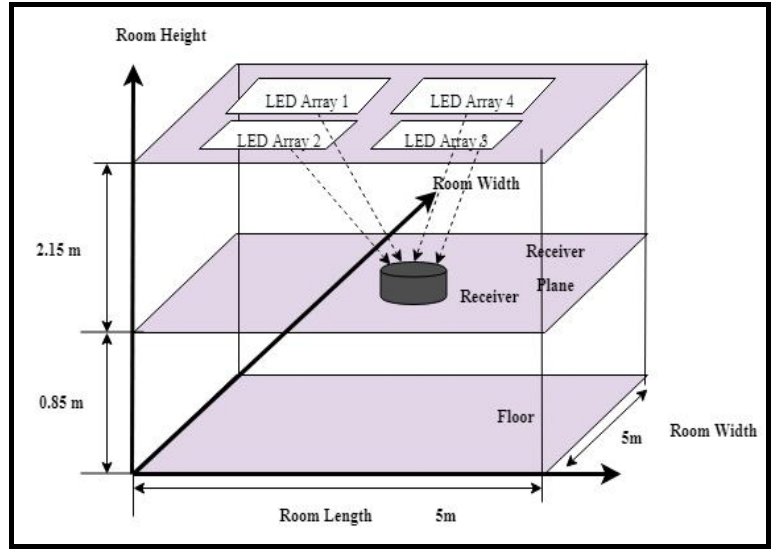

Figure 3: VLC with multiple LEDs in indoor environment

The simulation design consist of 3 parts, which are $\mathrm{Tx}$, channel and Rx.

Lambertian radiant intensity is featured in the LED is known as Lambert's emission law optics [10]. The law stated that the radiant intensity or luminous intensity observed from an ideal diffusely reflecting surface or ideal diffuse radiator is directly proportional to the cosine of the angle 0 between the direction of the incident light and the surface normal. The radiant intensity is defined as:

$$
R_{o}(\phi)=\lfloor(m+1) / 2 \pi\rfloor \cos ^{m}(\phi)
$$

The $\phi_{1 / 2}$ related to $m$ where it is the order of Lambertian emission, as $m=-\ln 2 / \ln \left(\cos \phi_{1 / 2}\right)$ is the Tx semi-angle located at half power. Both irradiance and incidence angle are $\phi$ and $\psi$. Whereas, the $P_{I X}=R_{L E D} * R_{o}\{\phi)$ as the transmitted power.

In this study, we design a simulation with line-of-sight (LOS) channel, where there is no obstruction between $\mathrm{Tx}$ and Rx. LOS propagation path is considered when estimation of the DC gain is accurate (Zeng at el, 2008). The channel is formulated as below:

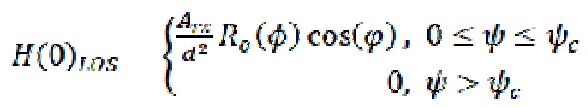

where the detector area is classified Arx, the distance between the Tx and the Rx is measured as d, the Tx radiant intensity R_o $(\phi)$ and the formula is given in Equation 1, the angle of incidence is $\varphi$, the FOV of the photodiode as $\square \varphi \rrbracket$ c.

The calculation of total power of $i$ LEDs in the directed path is in Equation 3

$$
P_{r x, L O S}=\sum_{i=1}^{L E D S} P_{t x} H_{R O S}^{i}(0)
$$

whereas $H_{i . a s}^{i}(0)$ is the $i t$ LED channel DC gain. 


\section{RESULTS AND DISCUSSION}

The performance of VLC with SISO and MISO is evaluated based on total receiving power. The received power is when the light passed through the optical filter and concentrator at the Rx.

$$
\text { P_rx }=(\text { P_LOS+P_diff }) * \operatorname{Tf}(\psi) * g(\psi)
$$

where $\operatorname{Tf}(\psi)$ the transmission coefficient of the optical is filter, and $\mathrm{g}(\psi)$ is the concentrator gain.

LED signal coverage is calculated based on Figure 4. The half angle of incidence of the simulation is maximum 30 degree between the Tx and Rx inside the room. Different half angle of incidences or LED viewing angle were tested by simulating the half angle of incidence from 5 degree to 30 degree.

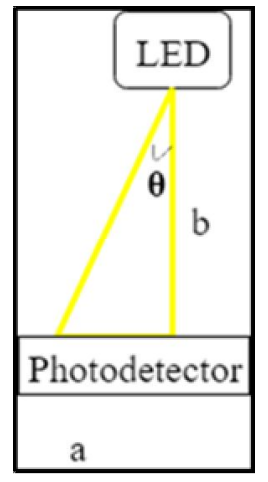

Figure 4: Radius of cell coverage calculation

The radius is theoretically determined using Equation 6 . This will calculate the LED signal coverage using the following equation,

$$
\tan \theta=\frac{u}{h}
$$

where $\tan \theta$ represent the half incident or irradiance angle, $\mathrm{b}$ refers as the height between the Tx and Rx and a as the radius coverage. To find the radius the equation looks like this:

$$
\mathrm{b} \times \tan \theta=a
$$

This equation will stimulate the radius coverage with the height between the Tx and $\mathrm{Rx}$ is using $1.5 \mathrm{~m}$. Each of the simulations are demonstrated using different half angle of incident in order to observe the changes which affected the LED signal coverage and the total received power.

Figure 5 shows received power for SISO in VLC performance when LED viewing angle is set to 5 degree. From Figure 5, it appears that the total received power is $-76 \mathrm{~dB}$ at the cell centre while the total received power is $-78 \mathrm{~dB}$ at the cell edge. It means that the stronger received power can be detected.

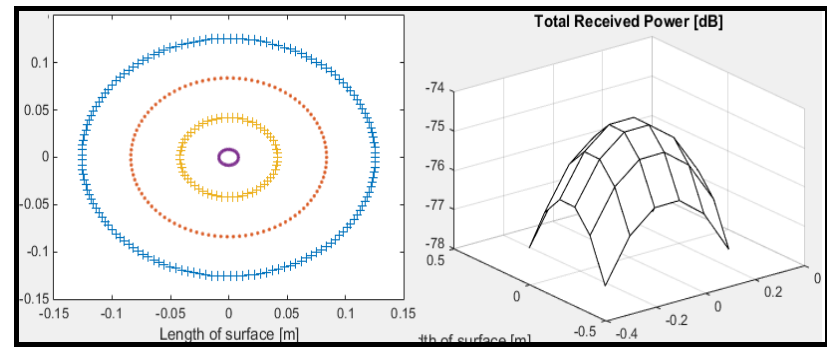

Figure 5: LED Viewing Angle/ Half angle of incidence at 5 degree

Figure 6 shows received power for SISO in VLC performance when LED viewing angle is set to 10 degree. From Figure 6, it is observed that the total received power at the cell centre is $-105 \mathrm{~dB}$ while it falls to $-125 \mathrm{~dB}$ at cell edge. The figure also depicts that the signal power received is stable in the center but it became weaker at the end of the coverage.

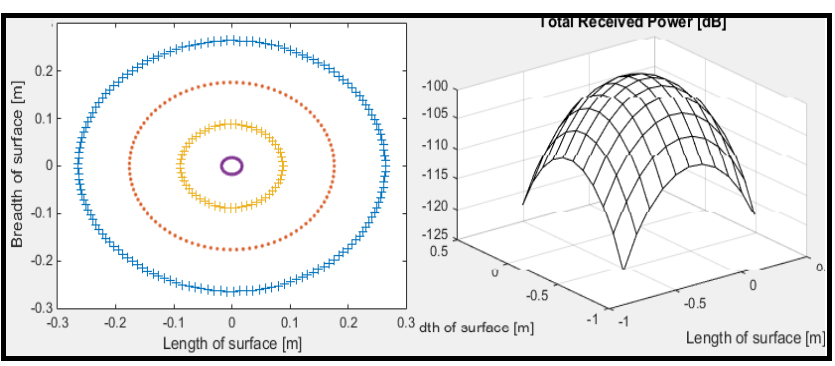

Figure 6: LED Viewing Angle/ Half angle of incidence at 10 degree

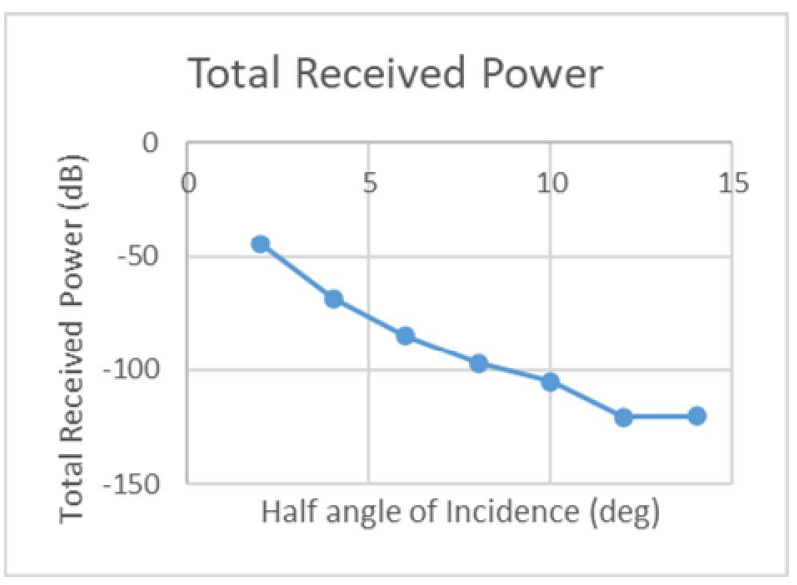

Figure 7: Total received power in SISO VLC at cell center

Figure 7 shows total received power for SISO in VLC at cell centre for different LED viewing angle. From Figure 7, it is observed that as the half angle of incidence increases as the total received power decreases. The maximum total received power achieved is $-44.18 \mathrm{~dB}$ at 2 degree while the minimum total received power would be $-120 \mathrm{~dB}$ at 14 degree. The LED signal's total received power at 14 degree gets fairly weaker even at the center.

The same pattern can be seen from Figure 8 when the performance is evaluated at cell edge, where the total received power decreases when LED viewing angle increases. We can see from the both graph that smaller LED viewing angle will 
have resulted to a higher total received power compared to larger LED viewing angle. For example, at 2 degree, $-44.18 \mathrm{~dB}$ power is detected at cell center and $-44.48 \mathrm{~dB}$ power is received at cell edge. At 14 degree, a lower power has been detected in the center $(-120 \mathrm{~dB})$ and cell edge $(-190 \mathrm{~dB})$. Thus, smaller viewing angle provide a stronger received power.

Figure 9 and 10 show total received power or SISO and MISO at 15 degree of LED viewing angle. For SISO, received power cannot be detected since the maximum LED viewing angle is set to 15 degree. However, from it clearly shown that even with 15 degree in MISO, the total received power is detected. Through the use of MISO, we can see how it affects the signal LED coverage as well as the total received power by applying multiple LEDs and multiple photodetectors despite the fact that the LED viewing angle is limited to a certain angle. After all, the simulation result shown in MISO clearly shows that by applying multiple LEDs, it can actually enhance the performance in LED power.

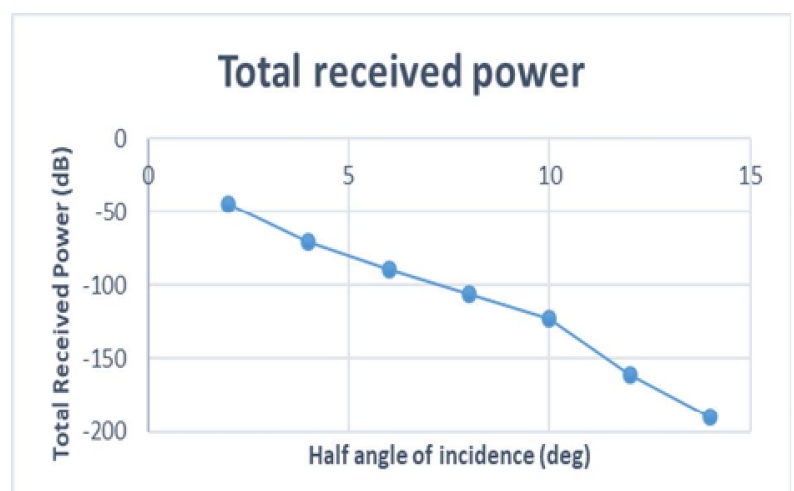

Figure 8: Total Received Power in SISO VLC at cell edge

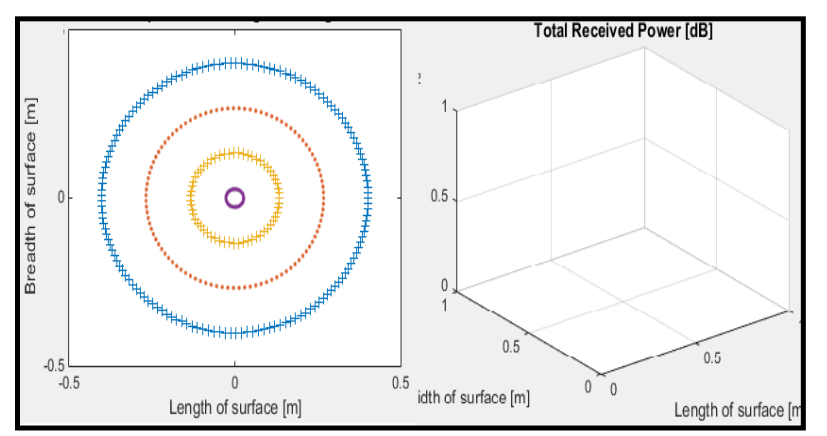

Figure 9: LED signal coverage and total received power at 15 degree in SISO

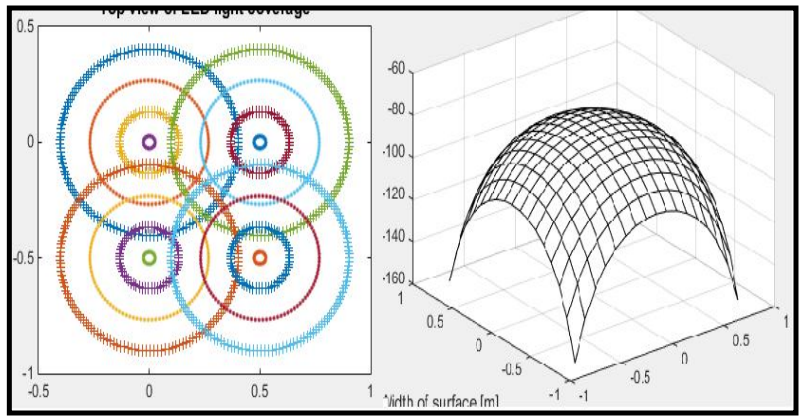

Figure 10 : LED signal coverage and total received power at 15 degree in MISO

\section{CONCLUSION}

The investigation of performance evaluation on SISO and MISO in VLC has been done clearly. The results of this research show that LED viewing angle affects the LED signal coverage and the total received power. The FOV in the photodetector also plays an important role to improve the received power at the photodiode significantly. It also been found that through MISO technique could improve performance in the visible light communication. This shown by demonstrating the half angle of incidence at 15 degree for both SISO and MISO. But due to a singular LED, the transmission of light to the Rx conclude with none total received power at all. However, with multiple LEDs has shown that the signal could still be received by the Rxs. In conclusion, MISO techniques could definitely improve the performance of a visible light communication.

\section{ACKNOWLEDGEMENT}

We would like to thank Research Group of Information Security Forensics and Computer Networking, Center for Advanced Computing Technology (C-ACT), Fakulti Teknologi Maklumat dan Komunikasi (FTMK), Universiti Teknikal Malaysia Melaka for support.

\section{REFERENCES}

1. W. A. Cahyadi, T. Jeong, Y. Kim, Y. Chung and T. Adiono. Patient monitoring using Visible Light uplink data transmission, IEEE International Symposium on Intelligent Signal Processing and Communication Systems (ISPACS), pp. 431-434, 2015.

https://doi.org/10.1109/ISPACS.2015.7432810

2. Mohamed, R. R., Mohamed, M. A., Azmi, K., Rao, E., \& Hashim, W. (2020). Indoor smart lighting controlling using human detection, International Journal of Advanced Trends in Computer Science and Engineering, vol. 9, no. 1.1 Special Issue, pp. 566-570. https://doi.org/10.30534/ijatcse/2020/7091.32020

3. Cesar A. L. and Elmer P. D. Development and Characterization of a Computer Vision System for Human Body Detection and Tracking under Low-light Condition, International Journal of Advanced Trends in Computer Science and Engineering, vol. 8, no. 2, pp. 251-254. https://doi.org/10.30534/ijatcse/2019/24822019

4. D. Karunatilaka, F. Zafar, V. Kalavally and R. Parthiban. LED Based Indoor Visible Light Communications: State of the Art, IEEE Communications Surveys \& Tutorials, vol. 17, no. 3, pp. 1649-1678, 2015.

5. T. Cevik and S. Yilmaz. (2015). An Overview Of Visible Light Communication System. International Journal of Computer Networks and Communications, vol.7, no. 6, pp.139-150, Nov 2015. 
6. R. Arya, N. Dailly and P. Kusumwal. A Performance Comparison of WiMAX-MIMO / MISO / SISO Communication Systems, Scientist Research Engineering Journal. 2010

7. J. Kumar, A. Patel, and S. Patel. Multiuser-MIMO Broadcast Channel techniques Multiuser-MIMO Broadcast Channel techniques, International Research Journal of Engineering and Technology (IRJET), vol. 3 no.2, pp.1587-1590, 2016.

8. R. K. Mondal, N. Saha and Y. M. Jang. Performance enhancement of MIMO based visible light communication. IEEE International Conference on Electrical Information and Communication Technology (EICT), pp. 1-5, Feb 2014 https://doi.org/10.1109/EICT.2014.6777901

9. W. Gu, and M. Aminikashani. Impact of Multipath Reflections on the Performance of Indoor Visible Light Positioning Systems, IEEE Journal of Lightwave Technology, vol. 34, no. 10, pp. 2578-2587, 15 May15, 2016.

10. M.V. Bhalerao1, M. Sumathi and S.S. Sonavane. Line of sight model for visible light communication using Lambertian radiation pattern of LED, International Journal of Communication Systems, vol.30, no.11, pp.

11. M. Mukherjee. Visible light communication-A survey of potential research challenges and advancements," IEEE 2017 Second International Conference on Electrical, Computer and Communication Technologies (ICECCT), pp. 1-8, 2017

https://doi.org/10.1109/ICECCT.2017.8117843

12. S. Fuada., A. Pratama Putra and T. Adiono. Analysis of Received Power Characteristics of Commercial Photodiodes in Indoor Los Channel Visible Light Communication. (IJACSA) International Journal of Advanced Computer Science and Applications vol.8, no. 7. pp. 164-172.

https://doi.org/10.14569/IJACSA.2017.080722 